HIV-Therapie

\title{
Bewertung des Nutzens muss neu justiert werden
}

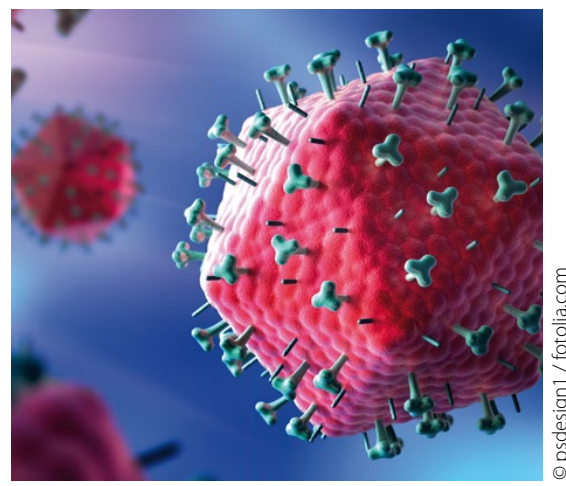

HIV-Viren - längst nicht mehr unmittelbar todbringend.

- Medizinische Fachgesellschaften bei der frühen Nutzenbewertung neuer Arzneien stärker zu berücksichtigen - das fordert die CDU-Gesundheitspolitikerin Karin Maag. „Darauf, wie die Expertise der Fachgesellschaften regelhaft in den AMNOG-Prozess eingebunden werden kann, müssen wir in der nächsten Legislaturperiode Antworten finden," so Maag. Die Besonderheit bei HIVMedikamenten sei, dass prospektive Studien mit der Dokumentation eines Zusatznutzens nicht möglich seien, wenn Endpunkte wie Überleben und aidsfreie Zeit keine Rolle mehr spielten, sagte Maag.

\section{Lebenserwartung HIV-Infizierter} annähernd so lang wie die Gesunder Der frühe Einsatz retroviraler Therapien hat dazu geführt, dass sich die Lebenserwartung HIV-infizierter Menschen an diejenige Nichtinfizierter annähert. Das Bundesgesundheitsministerium bezeichnet AIDS inzwischen als "gut behandelbare, chronische Erkrankung". Die Mehrheit der HIV-Patienten in Industriestaaten stirbt an Krankheiten, die nicht mit Aids zusammenhängen.

\section{Nebenwirkungen im Fokus}

Dass HIV-infizierte Menschen bei rechtzeitigem Beginn der Therapie sehr alt werden können, sei die Herausforderung bei der Entwicklung neuer Therapien und neuer Therapieregime. „Wir brauchen Medikamente mit weniger Nebenwirkungen," sagte Behrens. Die könnten helfen, die Adhärenz der Menschen zu stärken, die jahrzehntelang Tabletten einnehmen müssten. Zudem seien Kombipräparate gefragt, mit denen sich die komplexen Komorbiditäten HIV-infizierter Patienten behandeln ließen.

Behrens verwies darauf, dass die Zusammenarbeit zwischen niedergelassenen Ärzten und universitären Zentren eine Erfolgsgeschichte sei. $85 \%$ aller HIV-Therapien in Deutschland seien leitliniengerecht.

\section{- Anno Fricke}

Quelle: Symposium „HIV/Aids-Versorgung im Wandel: Wann nutzt der Fortschritt den Patienten?"; Berlin, Mai 2013 (Veranstalter: ViiV-Healthcare)

\section{Tumorschmerztherapie}

\section{Patienten profitieren von konstanter Schmerzkontrolle}

- Therapie der Wahl bei Tumorschmerzen ist nach der neuen Praxisleitline der Deutschen Gesellschaft für Schmerztherapie (DGS) (http://dgs-praxisleitlinien.de) die Pharmakotherapie mit oral zu applizierenden Substanzen der WHO-Stufen I bis III, erklärte Dr. Johannes Horlemann, Leiter des Regionalen Schmerzzentrums DGS Geldern und Vizepräsident der DGS. Transdermale Systeme können in fortgeschrittenen Tumorstadien Resorptionsstörungen unterliegen. Der Tumorpatient sollte keineswegs alle Stufen des WHO-Schemas durchschreiten müssen. Vorrang bei der Auswahl des Medikaments habe vielmehr die Wirkstärke und die Verträglichkeit.

\section{Dauertherapie den Vorzug geben}

Die Therapie sollte als Dauertherapie mit festen Einnahmezeiten so organisiert sein, dass möglichst eine 24-stündige Abdeckung durch eine Retardfreisetzung erreicht wird. Präferenzsubstanz in der Tumorschmerztherapie ist laut Praxisleitlinie Hydromorphon, das pharmakologische Vorteile in der Verträglichkeit aufweist und einem „idealen“ Medikament für die Tumorschmerztherapie recht nahe kommt (Tabelle 1). Das Stufe-III-Opioid wirkt gleichermaßen bei neuropathischen, nozizeptiven und viszeralen Schmerzen, so Horlemann.

Vorteilhaft ist Hydromorphon insbesondere in der OROS $^{\oplus}$-Galenik (Jurnista ${ }^{\circledR}$ ), die bei nur einmal täglicher Einnahme für eine kontrollierte, lang anhaltende Freisetzung sowie einen gleichmäßigen Plasmaspiegel über 24 Stunden sorgt. Das verlässliche Wirkzeitfenster von bis zu 24 Stunden vermeidet End-of-dose-Schmerzen [Güttler K, DAZ 2008;148:69-73]. Das durchgehende Freisetzungsprofil erzeugt einen flachen Plasmaspiegel, der zu einem besseren Verträglichkeitsprofil führt [Güttler $\mathrm{K}$ et al, Schmerz 2008;22(5):562-570].

\section{Tabelle}

Anforderungen an ein ideales Schmerzmedikament für die Tumorschmerztherapie

- Geringe Eiweißbindung (gute Steuerbarkeit)

- Keine (wenig) Interaktion über Cytochromsysteme

- Keine aktiven Metaboliten

- Steady state: $24 \mathrm{~h} / \mathrm{Tag}$

- Wenige Nebenwirkungen, insbesondere: keine Tagessedation

- Einfache Anwendung (Einmalgabe)

- Unterstützung des erholsamen Schlafes

- Dagmar Jäger-Becker

Quelle: Symposium "Gemeinsam gegen den (Tumor-)Schmerz" im Rahmen des Deutschen Schmerz- und Palliativkongresses, Frankfurt, März 2013 (Veranstalter: Janssen) 